\title{
INTERPRETABILITY DEGREES OF FINITELY AXIOMATIZED SEQUENTIAL THEORIES
}

\author{
ALBERT VISSER
}

\begin{abstract}
In this paper we show that the degrees of interpretability of finitely axiomatized extensions-in-the-same-language of a finitely axiomatized sequential theory - like Elementary Arithmetic EA, I $\Sigma_{1}$, or the Gödel-Bernays theory of sets and classes GB - have suprema. This partially answers a question posed by Vítěslav Švejdar in his paper [Šve78]. The partial solution of Švejdar's problem follows from a stronger fact: the convexity of the degree structure of finitely axiomatized extensions-in-the-same-language of a finitely axiomatized sequential theory in the degree structure of the degrees of all finitely axiomatized sequential theories.

In the paper we also study a related question: the comparison of structures for interpretability and derivability. In how far can derivability mimic interpretability? We provide two positive results and one negative result.
\end{abstract}

Dedicated to Dirk van Dalen on the occasion of his 80th birthday.

\section{INTRODUCTION}

In his paper [Šve78], Vítěslav Švejdar asks the following question. Suppose $A$ is a finitely axiomatized theory that interprets Robinson's Arithmetic Q. Let $\mathbb{V}_{A}$ be the partial ordering of the degrees of interpretability of the finitely axiomatized theories $B$ that are extensions ${ }^{1}$ of $A$. Is $\mathbb{V}_{A}$ a lattice? In particular, Švejdar asks whether $\mathbb{V}_{G B}$ is a lattice.

We solve this problem affirmatively for the case where $A$ is sequential. Švejdar's case of special interest GB is a sequential theory and, so, indeed, $\mathbb{V}_{G B}$ is a lattice. The suprema in $\mathbb{V}_{A}$, where $A$ is sequential, only coincide with the suprema in the lattice of all finitely axiomatized theories, in the trivial case where we take the supremum of theories that are comparable in the interpretability preordering. As we will see every lattice $\mathbb{V}_{A}$, for finitely axiomatized sequential $A$, is a distributive lattice.

Date: October 23, 2012.

I am grateful to Dick de Jongh and Paula Henk. This paper is part of a project shared with them. I thank Volodya Shavrukov for asking precisely the right questions and for his comments on an earlier manuscript of this paper. Special thanks are due to XS4ALL and the makers of Ghost. If not my recording of Ghost had, for mysterious reasons, stopped in the middle of the movie, I would not have been in the strange mood, somewhere between relaxation and irritation, that allowed me to see the proof of Theorem 6.1 as a matter of course. The next day the recording worked fine so that I could see the second half of the movie. Subsequently the paper grew around this proof.

${ }^{1}$ An extension will in this paper always be an extension in the same language. 
We also show that $\mathbb{V}_{Q}$ has suprema. In $\mathbb{V}_{Q}$ the suprema always coincide with the the suprema in the lattice of all finitely axiomatized theories.

The remaining cases are still wide open.

The existence of suprema both in the case of finitely axiomatized sequential theories and in the case of $Q$ follows from stronger facts. We show, in Section 6, that, for any finitely axiomatized sequential theory $A$, the embedding of the structure $\mathbb{V}_{A}$ into $\mathbb{D}_{\text {seq }}$, the degrees of finitely axiomatized sequential theories, is convex. This means that, whenever you have two finitely axiomatized sequential theories $A$ and $B$ with $A \triangleleft B$, there is a finite extension $C$ of $A$ with $C \equiv B$. Thus, for any finitely axiomatized sequential $A$, the structure $\mathbb{V}_{A}$ inherits the suprema of $\mathbb{D}_{\text {seq }}$.

Similarly, In the case of $Q$ we show, in Section 7 , that the embedding of $\mathbb{V}_{Q}$ into $\mathbb{D}_{\text {all }}$ the structure of the degrees of all finitely axiomatized theories is convex, i.e., for any finitely axiomatized theory $B$, if $\mathrm{Q} \triangleleft B$, there is a finitely axiomatized extension $C$ of $\mathrm{Q}$ such that $C \equiv B$. So the identical embedding functor of $\mathbb{V}_{\mathrm{Q}}$ into $\mathbb{D}_{\text {all }}$ is an isomorphism between $\mathbb{V}_{Q}$ and the cone above $Q$ in $\mathbb{D}_{\text {all }}$. Since $\mathbb{D}_{\text {all }}$ has suprema, so does $\mathbb{V}_{\mathrm{Q}}$.

In Section 4, we treat a closely related theme. How does interpretability compare to derivability? We will prove a number of results about embedding the degrees of interpretability of finitely axiomatized sequential theories into certain structures with the derivability ordering. The results of Section 6 will allow us to generalize one of the results, to wit Theorem 4.1, of Section 4. The generalization is Theorem 6.6. One could say that the results of Section 6 show that arbitrary finitely axiomatized sequential theories behave more like $S_{2}^{1}$ than one would think at first sight. This analogy can be best seen by comparing Theorem 3.4 and Theorem 6.5.

Remark 1.1. Why study the degrees of interpretability of extensions of a given theory? Is the point of interpretability not precisely independence of the tyranny of signature? Certainly, obtaining a coordinate-free view of theories is one of the applications of interpretations. However, categories of interpretations are more adequate to study this idea than degree structures for interpretability. For one thing, mutual interpretability is too crude as a plausible analysis of sameness of theories. Degrees of interpretability are more suitable to analyze the relative strength of theories. Given some theory the strength of principles extending the theory in the same language is of primary interest. E.g., both $\mathrm{ZF}+\mathrm{CH}$ and $\mathrm{ZF}+\neg \mathrm{CH}$ are interpretable in ZF, so $\mathrm{CH}$ is independent of ZF but does not add strength; this in clear distinction from large cardinal axioms.

\section{Preliminaries}

In this paper we will concentrate on finitely axiomatized theories of finite signature. We will view theories as given by a signature plus a specification of a set of sentences in the signature: the axioms.

We will assume that the finite signatures are given using a fixed finite alphabet. Thus, all structures we consider will be sets. The results of this paper can be reformulated for the stipulation that the finite signatures form a proper class, but we will refrain from doing this. 
A theory is finitely axiomatized when it is given by an enumeration of finitely many axioms of the form: $B$ is an axiom if $B=A_{0}$ or $\ldots, B=A_{n-1}{ }^{2}$ Par abus de langage, we will use $A, B, C \ldots$ as variables ranging both over sentences and over finitely axiomatized theories. This is pleasant since the names of the variables suggest the analogy with sentences. It is a bit misleading since there is a clear categorical distinction between a finitely axiomatized theory and a sentence. We use $T, U, V, \ldots$ for arbitrary theories.

We will use modal notations $\square_{U} A$ and $\diamond_{U} A$ for the (arithmetization of) $A$ is provable in $U$ and $U+A$ is consistent.

An $n$-proof is a proof from axioms with Gödel number smaller or equal than $n$ only involving formulas of complexity smaller or equal than $n$. To work conveniently with this notion, a good complexity measure $\rho$ is needed. This should satisfy three conditions. (i) Eliminating terms in favour of a relational formulation should raise the complexity only by a fixed standard number. (ii) Translation of a formula via the translation corresponding to an interpretation $K$ should raise the complexity of the formula by a fixed standard number depending only on $K$. (iii) The tower of exponents involved in cut-elimination should be of height linear in the complexity of the formulas involved in the proof.

Such a good measure of complexity $\rho$ together with a verification of desideratum (iii) - a form of nesting degree of quantifier alternations - is supplied in the work of Philipp Gerhardy. See [Ger03] and [Ger05]. It is also provided by Samuel Buss in his preliminary draft [Bus11]. Buss also proves that (iii) is fulfilled.

We will use $\square_{U, n} A$ for (the arithmetization of): $A$ is provable from $U$-axioms with Gödel numbers $\leq n$, where the formulas $B$ occurring in the proof satisfy $\rho(B) \leq n$. The formula $\diamond_{U, n} A$ means $\neg \square_{U, n} \neg A$.

In sequential theories we can define partial satisfaction predicates for formulas with complexity below $n$, for any $n$. The presence of these predicates has as a consequence that for any sequential theory $U$ and for any $n$, we can find an interpretation $N$ of a weak arithmetic like Buss' $\mathrm{S}_{2}^{1}$ in $U$ such that $U \vdash \diamond_{U, n}^{N} \top$. See e.g. [Vis93] for more details.

We refer the reader e.g. to [Vis11] or [Vis12] for the definitions of interpretation and interpretability.

We will consider degree structures $\mathbb{V}_{U}$ of finite extensions of the theory $U$. We will always use extension for: extension in the same language. We prefer to view the degree structure as a preorder category with as objects the theories given as signature plus specification of the axiom set and as morphisms arrows witnessing interpretability. We write the interpretability ordering as $\triangleleft$ and the induced equivalence as $\equiv$. So, $U \equiv V$ iff $U \triangleleft V$ and $V \triangleleft U$. The degrees are what one gets by dividing out the induced equivalence. We will employ both the preorder of theories way of viewing and the partial order of degrees way of viewing, using the qua locution. The reason we take the preorder perspective as basic is as follows.

\footnotetext{
${ }^{2}$ So, we do not count the theory $U$ that is given as follows as finitely axiomatized even if $d e$ facto is has finitely many axioms: $B$ is an axiom of $U$ if $B=\bigwedge \mathrm{Q}$ or, there is a $p$ smaller than the Gödelnumber of $B$ such that proof ${ }_{\mathrm{PA}}(p, \perp)$ and $B$ is an instance of the induction scheme. We consider $U$ as a perfectly legitimate theory, just not a finitely axiomatized one.
} 
We will consider mappings from theories $A$ to sentences $B_{A}$ that do not preserve interderivability. Thus, these constructions are intensional from the standpoint of the degrees and even of theories-viewed-as-sets of theorems.

We will also study degree structures of the form $\mathbb{D}_{P}$. These are the degrees of all finitely axiomatized theories having the structural property $P$. The two properties of special interest are seq, the property of being sequential, and all, the property of being any finitely axiomatized theory.

The default is that morphisms between degree structures are morphisms for the pre-order structure. If we want to consider e.g. lattice morphisms we will say so explicitly. We will say e.g. that two structures are isomorphic qua degree structures, when they are equivalent qua partial preorder categories. Etc.

\section{Degrees of Finitely Axiomatized Theories}

In this section we provide some well-known and basic results on the degrees of finitely axiomatized theories.

The structure of the degrees of all finitely axiomatized theories $\mathbb{D}_{\text {all }}$ is studied by Harvey Friedman in [Fri07]. He shows that this structure is a distributive lattice, that the degrees are dense, etc. We call the supremum of $A$ and $B$ in $\mathbb{D}_{\text {all }}: A \oplus B$. We call the infimum of $A$ and $B: A \otimes B$. Note that here we have specific realizations of the infimum and the supremum in mind. Thus, e.g., $\oplus$ maps a pair of theories to a theory. ${ }^{3}$

Remark 3.1. We note in passing that the structure of chapters (degrees of local interpretability of theories of arbitrary complexity and arbitrary (not necessarily finite) signature) studied in [MPS90] is isomorphic to the structure of the filters of $\mathbb{D}_{\text {all }}$ with $\subseteq$.

Since the emphasis in this paper is on the sequential case we review a number of basic facts about $\mathbb{D}_{\text {seq }}$, the structure of the degrees of finitely axiomatized sequential theories.

We first remind the reader of the definition of a sequential theory. An interpretation is direct when it is unrelativized and identity preserving. A theory is sequential if it directly interprets the theory Adjunctive Set Theory or AS. ${ }^{4}$

Adjunctive set theory AS is the following theory:

AS1. $\vdash \exists x \forall y \neg y \in x$,

AS2. $\vdash \forall u, v \exists x \forall y(y \in x \leftrightarrow(y \in u \vee y=v))$.

\footnotetext{
${ }^{3}$ Friedman assumes that we allow piece-wise interpretations. If we do not do that it suffices, in order to get the results stated in his lecture, to restrict ourselves to theories that prove that there are at least two elements, since piecewise interpretations can be simulated by more-dimensional ones as soon as we have two elements available. We note that the existence of suprema is not dependent on the assumption of two elements.

${ }^{4}$ We can allow more-dimensional direct interpretations. See [Vis10].
} 
The theory AS has a long history. See [Vis09]. For information on sequential theories, see e.g. [Pud83], [Pud85], [MPS90], [HP91] or [Vis10].

We can map an arbitrary theory $U$ to its minimal sequential extension $\mathrm{SEQ}(U)$ as follows. We first extend the language of $U$ by a new predicate $\Delta$. Then, we relativize all the quantifiers of the axioms of $U$ to $\Delta$, except the axioms for identity. We add the axiom that $\Delta$ is non-empty. Say, the resulting theory is $U^{\Delta}$. We extend the signature with a fresh binary predicate $\in$. Finally, we add the axioms of AS for $\in$. The necessity of relativization can be seen by contemplating the example of a theory saying that there are precisely three objects. See [Vis11] for more information about the functor SEQ. We have:

Theorem 3.2. Suppose emb is the identical embedding functor of $\mathbb{D}_{\text {seq }}$ into $\mathbb{D}_{\text {all }} .^{5}$ This is simply functor that maps $A$ to itself. Let $B$ be sequential. We have: $\mathrm{SEQ}(A) \triangleleft_{\text {seq }} B$ iff $A \triangleleft \operatorname{emb}(B)$. Thus, SEQ is the left adjoint of emb.

The theorem tells us that we can obtain a realization $A \sqcup B$ of the sequential supremum by taking SEQ $(A \oplus B) .{ }^{6}$ Thus, we have shown that there is a supremum in the sequential degrees. The sequential supremum $A \sqcup B$ is only equivalent to

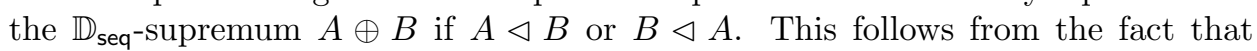
the sequential degrees are connected. This means that if some realization $E$ of the $\mathbb{D}_{\text {seq }}$-supremum of the degrees of $C$ and $D$ is sequential, then we must have $C \equiv E$ or $D \equiv E$. The connectedness of sequential theories was proved by Pavel Pudlák in [Pud83]. A different proof was given in [Ste89]. See also [MPS90].

The infimum $A \sqcap B$ of the finitely axiomatized sequential theories $A$ and $B$ can

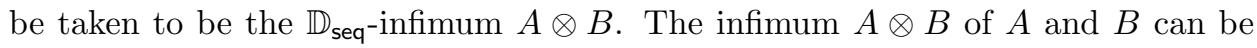
obtained by taking the union of the signatures of $A$ and $B$ and then taking the disjunction of the conjunctions of the axioms of $A$ resp. $B$.

We present a slightly different approach to the supremum in $\mathbb{D}_{\text {seq }}$. We have the following theorem.

Theorem 3.3. Suppose $A$ is finitely axiomatized and sequential. We have:

$$
A \equiv\left(\mathrm{S}_{2}^{1}+\diamond_{A, \rho(A)}^{\top}\right) \text {. }
$$

For a proof, see, [Pud85] or [HP91].

Now let $A$ and $B$ be finitely axiomatized and sequential. We claim that $C:=$ $\mathrm{S}_{2}^{1}+\diamond_{A, \rho(A)} \top+\diamond_{B, \rho(B)} \top^{\top}$ is a realization of the sequential supremum of $A$ and $B$. Clearly $C$ interprets both $A$ and $B$, by Theorem 3.3. Suppose $D \triangleright A$ and $D \triangleright B$. Then, for some $N$ and $M$ we have $N: D \triangleright\left(\mathrm{S}_{2}^{1}+\diamond_{A, \rho(A)}^{\top}\right)$ and $M$ : $D \triangleright\left(\mathrm{S}_{2}^{1}+\diamond_{B, \rho(B)} \top\right)$. By a theorem a Pavel Pudlák (see [Pud85]), there is an interpretation $K$ of $\mathrm{S}_{2}^{1}$ in $D$, such that there are definable initial embeddings of $K$ into both $N$ and $M$. By the downwards persistence of $\Pi_{1}$-sentences we will have both $\diamond_{A, \rho(A)}{ }^{\top}$ and $\diamond_{B, \rho(B)} \top$ on $K$. So $D \triangleright C$.

\footnotetext{
${ }^{5}$ We state the theorem for the specific degree structures we are interested in in this paper. In fact, the theorem extends to c.e. degrees and to arbitrary degrees.

${ }^{6}$ We note a notational awkwardness. In the order structures for relations like the subtheory relation and interpretability, the inconsistent theory is the top element. In Boole algebras falsum is the bottom. For this reason, e.g. the infimum $\sqcap$ corresponds to disjunction $\vee$.
} 
By similar reasoning, we obtain the following generalization. Let $A_{0}, \ldots, A_{n-1}$ be finitely axiomatized sequential theories. Then $\mathrm{S}_{2}^{1}+\bigwedge_{i<n} \diamond_{A_{i}, \rho\left(A_{i}\right)} \top$ is a realization of the sequential supremum of the $A_{i}$. It is easy to see that $S_{2}^{1}+\bigvee_{i<n} \diamond_{A_{i}, \rho\left(A_{i}\right)} \top$ is a realization of the sequential infimum of the $A_{i}$.

Thus we have shown:

Theorem 3.4. Let $A_{0}, \ldots, A_{n-1}$ be finitely axiomatized sequential theories. We have:

- $\left(\mathrm{S}_{2}^{1}+\bigwedge_{i<n} \diamond_{A_{i}, \rho\left(A_{i}\right)}^{\top}\right) \equiv \bigsqcup_{i<n} A_{i}$.

- $\left(\mathrm{S}_{2}^{1}+\bigvee_{i<n} \diamond_{A_{i}, \rho\left(A_{i}\right)}^{\top}\right) \equiv \prod_{i<n} A_{i}$.

The following is a fundamental result that relates interpretability to provability.

Theorem 3.5. For any $\Pi_{1}^{0}$-sentences $P, P^{\prime}$, we have:

$$
\left(\mathrm{S}_{2}^{1}+P\right) \triangleright\left(\mathrm{S}_{2}^{1}+P^{\prime}\right) \Leftrightarrow \mathrm{EA} \vdash P \rightarrow P^{\prime} .
$$

This theorem is due to Wilkie and Paris. See [WP87]. For a generalization, see: [Vis92]. ${ }^{7}$

We turn to a beautiful characterization of interpretability between finitely axiomatized sequential theories that is due to Harvey Friedman. See [Smo85].

Theorem 3.6. Suppose $A$ and $B$ are finitely axiomatized and sequential. ${ }^{8} W e$ have:

$$
A \triangleright B \quad \Leftrightarrow \quad \mathrm{EA} \vdash \diamond_{A, \rho(A)} \top \rightarrow \diamond_{B, \rho(B)} \top
$$

The proof is immediate by combining Theorem 3.3 and Theorem 3.5.

We end this section with two remarks about degree structures of finite extensions of a given theory.

Remark 3.7. We remind the reader that $\mathbb{V}_{W}$ is the degree structure of finite extensions of $W$. Suppose $U \dashv V$. What can we say about the relationship of $\mathbb{V}_{U}$ and $\mathbb{V}_{V}$ ? Clearly the identical embedding from $\mathbb{V}_{U}$ to $\mathbb{V}_{V}$ is a pre-order homomorphism. However, it is not necessarily convex and it is not necessarily a lattice homomorphism.

Consider for example $\mathrm{Q}$ and $\mathrm{PA}^{-}$, where we take the variant of $\mathrm{PA}^{-}$with the subtraction axiom. We have $\mathrm{Q} \dashv \mathrm{PA}^{-}$. The $\mathbb{V}_{\mathrm{PA}^{-}}$-supremum of two incomparable extensions $A$ and $B$ of $\mathrm{PA}^{-}$is sequential, but the $\mathbb{V}_{\mathrm{Q}^{-}}$supremum of $A$ and $B$ is not sequential. See Section 7 . It follows from this that the embedding is not convex.

Remark 3.8. Is there a good notion of sameness of theories that preserves the degree structures above those theories via an effective isomorphism? We show that the degree structures are thus preserved by sentential congruence.

\footnotetext{
${ }^{7}$ We find the theorem also formulated with $\mathrm{Q}, \mathrm{PA}^{-}$and $\mathrm{I} \Delta_{0}+\Omega_{1}$ in the role of $\mathrm{S}_{2}^{1}$. It is easy to see that all these versions are equivalent.

${ }^{8}$ Inspection of the proof shows that only $A$ needs to be sequential.
} 
Two theories $U$ and $V$ are sententially congruent if there are interpretations $K$ : $U \triangleleft V$ and $M: V \triangleleft U$ such that, for all $C$ in the language of $U$ and for all $D$ in the language of $V$, we have $U \vdash C \leftrightarrow C^{K M}$ and $V \vdash D \leftrightarrow D^{M K}$. We note that sentential congruence identifies more theories that e.g. bi-interpretability. ${ }^{9}$

Consider two theories $U$ and $V$. The only restriction we place on $U$ and $V$ is that they are of finite signature. We allow theories of arbitrary complexity. We suppose the finite extensions of e.g. $U$ are simply given by sentences axiomatizing them over $U$. In other words, since $U$ is the fixed background theory, we omit its axioms. We treat extensions of $V$ similarly.

Suppose $K$ and $M$ are a sentential congruence between $U$ and $V$. We consider the mappings $\Phi:(U+C) \mapsto\left(V+C^{K}\right)$ and $\Psi:(V+D) \mapsto\left(U+D^{M}\right)$. Par abus de langage we confuse $K$ and $M$ with their extended versions between stronger theories. We have:

$K:(U+C) \triangleleft\left(V+C^{K}\right)$ and $M:\left(V+C^{K}\right) \triangleleft\left(U+C^{K M}\right)$ and $\left(U+C^{K M}\right) \dashv(U+C)$. So $U+C$ is sententially congruent with $V+C^{K}$ and, similarly $V+D$ is sententially congruent with $U+D^{M}$. It follows that if $(U+C) \triangleleft\left(U+C^{\prime}\right)$, then:

$$
\Phi(U+C)=\left(V+C^{K}\right) \equiv(U+C) \triangleleft\left(U+C^{\prime}\right) \equiv\left(V+C^{\prime K}\right)=\Phi\left(U+C^{\prime}\right),
$$

and similarly for $\Psi$. So $\Phi$ and $\Psi$ are pre-order morphisms.

Finally, $\Psi(\Phi(U+C))=\left(U+C^{K M}\right) \dashv(U+C)$, so, a fortiori, $\Psi(\Phi(U+C)) \equiv$ $(U+C)$, and similarly for $\Phi \Psi$.

We may conclude that $\Phi$ and $\Psi$ form an equivalence of categories between $\mathbb{V}_{U}$ and $\mathbb{V}_{V}$.

\section{Derivability And Interpretability}

In this section we compare the degrees of finitely axiomatized sequential theories $\mathbb{D}_{\text {seq }}$ with structures $\mathbb{P}_{A}$ of finitely axiomatized extensions of $A$ with the derivability ordering $\dashv$, for sequential $A$.

The following list of properties provides a loose framework for the results of this section. Suppose we have a map $\Theta$ from the finitely axiomatized sequential theories to the finitely axiomatized sequential theories of signature $\Sigma^{\star}$. Suppose further we have a, possibly partial, map $\Psi$ on the finitely axiomatized sequential theories of signature $\Sigma^{\star}$ to the finitely axiomatized sequential theories that is at least defined on the image of $\Theta$. We demand:

- if $A \triangleleft B$, then $\Theta(A) \dashv \Theta(B)$.

- if $A \dashv B$, then $\Psi(A) \triangleleft \Psi(B)$, whenever $A$ and $B$ are in the domain of of $\Psi$.

- $\Psi(\Theta(A)) \equiv A$.

- $\Psi$ is recursive.

We consider the following properties:

I. $\Psi$ is given by the identical mapping $A \mapsto A$.

\footnotetext{
${ }^{9}$ In fact there is a verified separating example due to Harvey Friedman.
} 
II. for some $k$, and for all finitely axiomatized sequential $A, \rho(\Theta(A)) \leq k$.

III. $\Theta$ is recursive.

We will show that properties (I) and (II) are satisfiable (Theorem 4.1). We prove that properties (II) and (III) are satisfiable (Theorem 4.4). Properties (I), (II), (III) are not satisfiable (Theorem 4.5). I do not know whether (I) and (III) are satisfiable.

Theorem 4.1. There is a function $\theta$ that sends finitely axiomatized sequential theories $A$ to $\forall \Pi_{1}^{\mathrm{b}}$-sentences such that:

- if $A \triangleright B$, then $\mathrm{S}_{2}^{1}+\theta(A) \vdash \mathrm{S}_{2}^{1}+\theta(B)$.

- $A \equiv\left(\mathrm{S}_{2}^{1}+\theta(A)\right)$.

The function $\theta$ is recursive in an oracle for a $\Sigma_{1}$-complete set.

We see that (I) and (II) are fulfilled by taking $\Theta(A):=\mathrm{S}_{2}^{1}+\theta(A)$ and $\Psi(A):=A$.

The proof of Theorem 4.1 is an exercise in propositional logic.

Proof. Let $\left(A_{i}\right)_{i \in \omega}$ be an enumeration without repetitions of the finitely axiomatized sequential theories. We choose our enumeration in such a way that it is recursive. ${ }^{10}$ We define:

- $C_{i}:=\bigwedge\left\{\diamond_{\rho\left(A_{j}\right), A_{j}} \top \mid j \leq i\right.$ and $\left.A_{i} \triangleright A_{j}\right\}$,

- $B_{k}:=\bigvee\left\{C_{\ell} \mid \ell \leq k\right.$ and $\left.A_{\ell} \triangleright A_{k}\right\}$

We note that the definition of the $C_{i}$ and $B_{k}$ requires an oracle for a complete recursively enumerable set.

Suppose $A_{m} \triangleright A_{n}$. We show that $B_{m} \vdash B_{n}$. Suppose $C_{i}$ is any disjunct of $B_{m}$. We have $i \leq m$ and $A_{i} \triangleright A_{m} \triangleright A_{n}$. If $i \leq n$, then $C_{i}$ will also be a disjunct of $B_{n}$. Suppose $i>n$. We show that $C_{i} \vdash C_{n}$. For any conjunct $\diamond_{A_{j}, \rho\left(A_{j}\right)} \top$ of $C_{n}$, we have $j \leq n$ and $A_{n} \triangleright A_{j}$, hence $j \leq i$ and $A_{i} \triangleright A_{j}$. So $\diamond_{A_{j}, \rho\left(A_{j}\right)}{ }^{\top}$ is a conjunct of $C_{i}$. Ergo, each disjunct $C_{i}$ of $B_{m}$ implies a disjunct of $B_{n}$. We may conclude that $B_{m} \vdash B_{n}$.

We find:

$$
\begin{aligned}
\mathrm{S}_{2}^{1}+C_{i} & =\mathrm{S}_{2}^{1}+\bigwedge\left\{\diamond_{\rho\left(A_{j}\right), A_{j}}^{\top} \mid j \leq i \text { and } A_{i} \triangleright A_{j}\right\} \\
& \equiv \bigsqcup\left\{A_{j} \mid j \leq i \text { and } A_{i} \triangleright A_{j}\right\} \\
& \equiv A_{i} \\
\mathrm{~S}_{2}^{1}+B_{k} & =\mathrm{S}_{2}^{1}+\bigvee\left\{C_{\ell} \mid \ell \leq k \text { and } A_{\ell} \triangleright A_{k}\right\} \\
& \equiv \prod\left\{\mathrm{S}_{2}^{1}+C_{\ell} \mid \ell \leq k \text { and } A_{\ell} \triangleright A_{k}\right\} \\
& \equiv \prod\left\{A_{\ell} \mid \ell \leq k \text { and } A_{\ell} \triangleright A_{k}\right\} \\
& \equiv A_{k}
\end{aligned}
$$

\footnotetext{
${ }^{10}$ If we choose our enumeration with repetitions $\theta$ will only be functional modulo mutual derivability of the values, which seems to be not such a bad thing.
} 
We take $\theta\left(A_{i}\right):=B_{i}$.

Remark 4.2. In the proof of Theorem 4.1, we could use the following alternative definition: $C_{i}:=\diamond_{\rho\left(A_{n_{i}}\right), A_{n_{i}}} \top$, where $n_{i}$ is chosen to be sufficiently large w.r.t. the $n_{j}$, for $j<i$, and the complexities of the least complex interpretations witnessing $A_{i} \triangleright A_{j}$, for $j<i$.

Remark 4.3. Let the identical projection $\pi: \mathbb{P}_{\mathrm{S}_{2}^{1}} \rightarrow \mathbb{D}_{\text {seq }}$ be given by $\pi(A):=A$. Let $\Theta: \mathbb{D}_{\text {seq }} \rightarrow \mathbb{P}_{\mathrm{S}_{2}^{1}}$ be given by $\Theta(A):=\mathrm{S}_{2}^{1}+\theta(A)$. When we think modulo induced equivalence relations, Theorem 4.1 tells us that $\pi$ is a retraction, where $\Theta$ is the witnessing coretraction.

We consider the extensions of EA with a $\Pi_{1}$-sentence ordered by $\dashv$. Let's call this structure $\mathbb{P}_{E A}^{\Pi_{1}}$. (In the terminology of [LS08], the lattice corresponding to $\mathbb{P}_{E A}^{\Pi_{1}}$ would be designated by $\Pi_{1} / E A$.)

Theorem 4.4. The degrees of interpretability of finitely axiomatized sequential theories are recursively equivalent to the degrees of derivability of $\Pi_{1}^{0}$-sentences over EA. In other words, $\mathbb{D}_{\text {seq }}$ is recursively equivalent to $\mathbb{P}_{\mathrm{EA}}^{\Pi_{1}}$.

Clearly, Theorem 4.4 tells us that conditions (II) and (III) are satisfiable. In fact the result gives us more than that.

Proof. Let $\Theta: A \mapsto \mathrm{EA}+\diamond_{A, \rho(A)} \top^{\top}$ and $\Psi: \mathrm{EA}+P \mapsto \mathrm{S}_{2}^{1}+P .{ }^{11}$

By Theorem 3.6, $\Theta$ is a morphism. By Theorem 3.5, $\Psi$ is a morphism.

We note that $\Psi(\Theta(A)):=\mathrm{S}_{2}^{1}+\diamond_{A, \rho(A)} \top$. So, by Theorem 3.3, $\Psi(\Theta(A)) \equiv A$.

Moreover $\Theta(\Psi(\mathrm{EA}+P))=\mathrm{EA}+\diamond_{\mathrm{S}_{2}^{1}, \rho\left(\mathrm{S}_{2}^{1}+P\right)} P$. Since we have $\mathrm{EA} \vdash \diamond_{\mathrm{S}_{2}^{1}, \rho\left(\mathrm{S}_{2}^{1}\right)} \top$, we find by standard reasoning that EA $\vdash \diamond_{\mathrm{S}_{2}^{1}, \rho\left(\mathrm{S}_{2}^{1}+P\right)} P \leftrightarrow P$. Hence, we may conclude that $\Theta(\Psi(\mathrm{EA}+P)) \dashv(\mathrm{EA}+P)$.

Theorem 4.4 together with the specification of the isomorphism can be viewed as the proper form of the Friedman characterization. It is extremely useful. All kinds of properties that are known for $\mathbb{P}_{E A}^{\Pi_{1}}$ can be transferred to $\mathbb{D}_{\text {seq. }}$. For example, it is a consequence of Theorem 3.1 of [Sha10] that the first-order theory of $\mathbb{D}_{\text {seq }}$ (qua degree structure) is not arithmetical. The same holds for any of its non-trivial intervals. Theorem 6.1 of this paper tells us that, for any consistent, finitely axiomatized and sequential theory $A$, the structure $\mathbb{V}_{A}$ is (isomorphic to) a non-trivial interval. So, e.g. the first-order theories of $\mathbb{V}_{\mathrm{PA}^{-}}$and $\mathbb{V}_{\mathrm{I} \Sigma_{1}}$ and $\mathbb{V}_{\mathrm{GB}}$ (qua degree structures) are not arithmetical. By Theorem 0.1 of [LS08], the $\forall \exists$-theory of $\mathbb{D}_{\text {seq }}$ is decidable.

We turn to the promised negative result.

Theorem 4.5. Let $\Theta$ be a function from arbitrary finitely axiomatized sequential theories to finitely axiomatized sequential theories of a fixed signature $\Sigma^{\star}$. The following list of properties cannot be simultaneously fulfilled

a. if $A \triangleright B$, then $\Theta(A) \vdash \Theta(B)$.

b. $A \equiv \Theta(A)$.

\footnotetext{
${ }^{11}$ Note that here our choice to consider theories as given by axioms is essential.
} 
c. There is a number $n$ such that, for all finitely axiomatized sequential $A$, we have $\rho(\Theta(A)) \leq n$.

d. $\Theta$ is recursive.

We note that $(\mathrm{d})$ is equivalent to the demand that $\Theta$ is a partial recursive function that is defined on the indices of finitely axiomatized sequential theories. This can be seen by using the functor SEQ.

Proof. Suppose $\Theta$ satisfies (a)-(d).

Let $A_{0}:=\Theta\left(\mathrm{S}_{2}^{1}\right)$. Clearly, $A_{0} \dashv \Theta(A)$, for any finitely axiomatized sequential $A$. Let $n$ be a bound on the complexity of the $\Theta(A)$ and let $f$ be the recursive function implementing $\Theta$.

We claim that there is a $\Sigma^{\star}$-formula $C x$ such that, for all finitely axiomatized sequential $A, \Theta(A) \dashv \vdash C\left\ulcorner\right.$. We can find an $N: \mathrm{S}_{2}^{1} \triangleleft A_{0}$ such that there is a satisfaction predicate for formulas of complexity below $n$ which are coded in $N$. Let $\mathrm{True}_{n}$ be the truth-predicate derived from Sat ${ }_{n}$. Let $F x y$ represent the function $f$ in $A_{0}$ w.r.t. the $N$-numbers. We can take:

$$
C(x):=A_{0} \wedge \exists y \in \delta_{N}\left(F x y \wedge \operatorname{True}_{n}(y)\right) .
$$

We write $C(A)$ for $C(\ulcorner A\urcorner)$.

By the Gödel Fixed Point Lemma, we can find a sentence $B$ such that

$$
A_{0} \vdash B \leftrightarrow \neg C\left(A_{0} \wedge B\right)
$$

We have: $\left(A_{0} \wedge B\right) \equiv C\left(A_{0} \wedge B\right) \equiv\left(A_{0} \wedge \neg B\right)$. Since both $\left(A_{0} \wedge B\right) \triangleright\left(A_{0} \wedge B\right)$ and $\left(A_{0} \wedge \neg B\right) \triangleright\left(A_{0} \wedge B\right)$, we find that $A_{0} \triangleright\left(A_{0} \wedge B\right)$. It follows that $C\left(A_{0}\right) \vdash C\left(A_{0} \wedge B\right)$. We also have $A_{0} \equiv \mathrm{S}_{2}^{1}$, and hence $C\left(A_{0}\right) \dashv C\left(\mathrm{~S}_{2}^{1}\right)=A_{0}$. Ergo $A_{0} \vdash C\left(A_{0} \wedge B\right)$ and thus $A_{0} \vdash \neg B$. It follows that $A_{0} \triangleright\left(A_{0} \wedge B\right) \vdash \perp$. Hence $A_{0}$ is inconsistent and so is $\mathrm{S}_{2}^{1}$. Quod non.

Open Question 4.6. Are (I) and (III) of the list at the beginning of this section satisfiable?

\section{FGH Arguments}

In this section we prove FGH-style results. ${ }^{12}$ The FGH Theorem is a superior way to set things right even if the world is fundamentally wrong: the main conditions are (i) that the Creatrix plays first and (ii) that She builds a consistent world. For information about the FGH Theorem, see e.g. [Vis05].

We remind the reader of witness comparison notation. Suppose $C=\exists x C_{0}(x)$ and $D=\exists y D_{0}(y)$. We define:

- $C \leq D: \Leftrightarrow \exists x\left(C_{0}(x) \wedge \forall y<x \neg D_{0}(y)\right.$.

- $D<C: \Leftrightarrow \exists x\left(D_{0}(x) \wedge \forall y \leq x \neg C_{0}(y)\right.$.

\footnotetext{
${ }^{12}$ FGH stands for Friedman-Goldfarb-Harrington. The order of names does not reflect the temporal order of discovery, of which I am not entirely sure.
} 
- Suppose $E=(C \leq D)$, then $E^{\perp}:=(D<C)$.

- Suppose $E=(D<C)$, then $E^{\perp}:=(C \leq D)$.

The versions of the FGH Theorem proved in Theorem 5.1 below are quite close to the original one, the only variation being the use of restricted provability.

Theorem 5.1. Let $A$ be a finitely axiomatized theory. Suppose $N: \mathrm{S}_{2}^{1} \triangleleft A$. Let $S$ be any $\Sigma_{1}$-sentence. ${ }^{13}$ Let $n$ be bigger than or equal to the maximum of $\rho(A), \rho(S)$, $\rho\left(\operatorname{prov}_{A}(x)\right)+\rho(N)+3$. Then, there are $\Sigma_{1}$-sentences $R_{0}$ and $R_{1}$ such that:

$$
\begin{aligned}
& \text { i. EA } \vdash \square_{A, n} R_{0}^{N} \leftrightarrow\left(S \vee \square_{A, n} \perp\right) . \\
& \text { ii. } \mathrm{EA} \vdash \square_{A, n} \neg R_{1}^{N} \leftrightarrow\left(S \vee \square_{A, n} \perp\right) .
\end{aligned}
$$

Proof. We first prove (i). We find $R_{0}$ such that:

$$
\mathrm{S}_{2}^{1} \vdash R_{0} \leftrightarrow S \leq \square_{A, n} R_{0}^{N} .
$$

Note that $n$ is chosen in such a way that $S \leq \square_{A, n} R_{0}^{N}$ makes sense. We show that EA $\vdash \square_{A, n} R_{0}^{N} \leftrightarrow\left(S \vee \square_{A, n} \perp\right)$. Reason in EA.

Suppose $\square_{A, n} R_{0}^{N}$. It follows that $R_{0}$ or $R_{0}^{\perp}$. In the first case, we have, by the fixed point property of $R_{0}$, that $S$. In the second case we have, by $\Sigma_{1}$-completeness, $\square_{A, n}\left(R_{0}^{\perp}\right)^{N}$, and hence $\square_{A, n} \perp$.

Conversely, suppose $S$. It follows that $R_{0}$ or $R_{0}^{\perp}$. In the first case it follows, by $\Sigma_{1}$ completeness that $\square_{A, n} R_{0}^{N}$. In the second case it follows, by fixed point property of $R_{0}^{\perp}$, that $\square_{A, n} R_{0}^{N}$. Finally, if we have $\square_{A, n} \perp$, we immediately have $\square_{A, n} R_{0}^{N}$.

We prove (ii). We find $R_{1}$ such that:

$$
\mathrm{S}_{2}^{1} \vdash R_{1} \leftrightarrow \square_{A, n} \neg R_{1}^{N} \leq S .
$$

We show that EA $\vdash \square_{A, n} \neg R_{1}^{N} \leftrightarrow\left(S \vee \square_{A, n} \perp\right)$. Reason in EA.

Suppose $\square_{A, n} \neg R_{1}^{N}$. It follows that $R_{1}$ or $R_{1}^{\perp}$. In the first case, we have, by $\Sigma_{1^{-}}$ completeness, $\square_{A, n} R_{1}^{N}$ and hence $\square_{A, n} \perp$. In the second place, we have by the fixed point property of $R_{1}$, that $S$.

Conversely, suppose $S$. It follows that $R_{1}$ or $R_{1}^{\perp}$. In the first case it follows, by the fixed point property of $R_{1}$, that $\square_{A, n} \neg R_{1}^{N}$. In the second case, we have, by $\Sigma_{1}$ completeness, that $\square_{A, n}\left(R_{1}^{\perp}\right)^{N}$, and hence $\square_{A, n} \neg R_{1}^{N}$. Finally, if we have $\square_{A, n} \perp$, we immediately have $\square_{A, n} \neg R^{N}$.

The proofs of Theorem 6.1 and 6.4 use only Theorem 5.1. So the reader could skip Theorem 5.2 for the moment and first study Theorems 6.1 and 6.4.

The following theorem FGH-style theorem is a variant and refinement of a sequence of FGH theorems proved in [Vis93], [Vis05] and [Vis12]. The first two papers prove versions of theorems due Jan Krajíček (see [Kra87]) and Harvey Friedman (see [Smo85]). Krajíček's work is based on results from Alex Wilkie's fundamental paper [Wil86].

\footnotetext{
${ }^{13}$ Note that we do not need that $A$ is sequential.
} 
Theorem 5.2. Let $A$ be a finitely axiomatized sequential theory. Let $k$ be any number. We can find an interpretation $N_{0}: \mathrm{S}_{2}^{1} \triangleleft A$, such that, for every $\Sigma_{1}$ sentence $S$ with $\rho(S) \leq k$ :

$$
\mathrm{EA} \vdash \square_{A, m} S^{N_{0}} \leftrightarrow\left(S \vee \square_{A, \rho(A)} \perp\right) .
$$

Here $m:=\max \left(\rho(A), k+\rho\left(N_{0}\right)\right)$.

Proof. Let $A$ be finitely axiomatized and sequential. Suppose $N: \mathrm{S}_{2}^{1} \triangleleft A$. Let $k$ be any number. Consider any $\Sigma_{1}$-sentence $S$ with $\rho(S) \leq k$.

Let $n:=\max \left(\rho\left(\operatorname{prov}_{A, x}(y)\right)+1, k+\rho(N)\right)$. Let $R$ be chosen such that $\mathrm{S}_{2}^{1} \vdash R \leftrightarrow$ $S \leq \square_{A, n} R^{N}$. Note that $n$ is large enough such that $\square_{A, n} R^{N}$ makes sense. We note that the choice of $n$ only depends on $A, N$ and $k$. We can find a cut $N_{0}$ of $N$ such that, in $A$, we have the minimum principle for $\Delta_{0}\left(\omega_{1}\right)$-formulas of complexity less than or equal to $k$ and such that $A \vdash \forall x\left(\operatorname{prov}_{A, n}^{N_{0}}(x) \rightarrow \operatorname{True}_{n}(x)\right)$. We have:

$$
\begin{aligned}
A \vdash S^{N_{0}} & \rightarrow\left(R \vee R^{\perp}\right)^{N_{0}} \\
& \rightarrow R^{N} \vee\left(\square_{A, n} R^{N}\right)^{N_{0}} \\
& \rightarrow R^{N}
\end{aligned}
$$

Let $m$ be $\max \left(\rho(A), k+\rho\left(N_{0}\right)\right)$. We have:

$$
\begin{aligned}
\mathrm{EA} \vdash \square_{A, m} S^{N_{0}} & \rightarrow \square_{A, p} R^{N} \\
& \rightarrow \square_{A, n} R^{N} \\
& \rightarrow R \vee R^{\perp} \\
& \rightarrow S \vee\left(R^{\perp} \wedge \square_{A, n} R^{N}\right) \\
& \rightarrow S \vee \square_{A, n} \perp \\
& \rightarrow S \vee \square_{A, \rho(A)} \perp
\end{aligned}
$$

Here Step 1 uses our previous insight. The number $p$ is a sufficiently large standard number. Step 2 uses the fact that in EA we have cut-elimination for cuts of standard complexity. Similarly for Step 6.

Conversely, we clearly have: EA $\vdash\left(S \vee \square_{A, \rho(A)} \perp\right) \rightarrow \square_{A, n} S^{N_{0}}$.

We note that the statement of the theorem and the construction of $N_{0}$ are entirely self-reference free. It is just the proof that uses the FGH-style Rosser-construction. This makes one wonder whether there is also a self-reference-free proof.

\section{Convexity}

Let $A$ be a finitely axiomatized sequential theory. We prove the convexity of the embedding of $\mathbb{V}_{A}$ into $\mathbb{D}_{\text {seq }}$.

We note that this is easy if $A=\mathrm{S}_{2}^{1}$. Suppose $B$ is finitely axiomatized and sequential. We have, by Theorem 3.3, that, for sufficiently large $n, B \equiv\left(\mathrm{S}_{2}^{1}+\diamond_{B, n} \top\right)$. We will prove Theorem 6.5 as an analogue of Theorem 3.4 . Theorem 6.5 has the desired analogue of Theorem 3.3 as a direct consequence. Thus any finitely axiomatized sequential theory $A$ behaves in certain respects as $\mathrm{S}_{2}^{1}$. 
In Theorem 6.1 we will follow a more direct and simpler approach to prove the desired convexity result.

Theorem 6.1. Let $A$ be a finitely axiomatized and sequential. Let $N: A \triangleright \mathrm{S}_{2}^{1}$. Suppose $B \triangleright A$. Then there is a $\Pi_{1}$ sentence $Q$ and $a \Sigma_{1}$ sentence $R$, such that (i) $B \equiv\left(A+Q^{N}\right)$ and (ii) $B \equiv\left(A+R^{N}\right)$.

More precisely, we can take $Q$ to be $\forall \Pi_{1}^{\mathrm{b}}$ and $R$ to be $\exists \Sigma_{1}^{\mathrm{b}}$.

Theorem 6.1 tells us that the identical embedding of $\mathbb{V}_{A}$, where $A$ is finitely axiomatized and sequential, into $\mathbb{D}_{\text {seq }}$ is convex, and, ipso facto, a lattice homomorphism.

Proof. We assume the conditions of the theorem. Suppose $n$ is sufficiently large. It follows that

$$
\mathrm{EA} \vdash \diamond_{B, n} \top \rightarrow \diamond_{A, n} \top .
$$

Let $Q:=\neg R_{0}$, where $R_{0}$ is as in Theorem 5.1(i), with $S:=\square_{B, n} \perp$. We find:

$$
\begin{aligned}
\mathrm{EA} \vdash \diamond_{A, n} Q^{N} & \leftrightarrow\left(\diamond_{B, n} \top \wedge \diamond_{A, n} \top\right) \\
& \leftrightarrow \diamond_{B, n} \top .
\end{aligned}
$$

Here Step 8 is just the contraposition of Theorem 5.1(i) and Step 9 is by Equation 7. It follows that:

$$
\begin{aligned}
B & \equiv \mathrm{S}_{2}^{1}+\diamond_{B, n} \top \\
& \equiv \mathrm{S}_{2}^{1}+\diamond_{A, n} Q^{N} \\
& \equiv A+Q^{N} .
\end{aligned}
$$

Here Steps 10 and 12 are by Theorem 3.3 and Step 11 is by Equations 8 and 9 in combination with Theorem 3.5.

The proof of (ii) is similar applying Theorem 5.1 with $S:=\square_{B, n} \perp$ and setting $R:=R_{1}$.

Remark 6.2. It is not difficult to eliminate the use of Theorem 3.5 from the proof of Theorem 6.1. For example let $I$ be an $S_{2}^{1}$-cut such that $S_{2}^{1} \vdash \forall x \in I \exists y 2^{2^{x}}=y$ and such that we have $\mathrm{T}_{2}^{1}$ on $I$. Then we can prove in a direct way, with $R_{0}$ as in the proof of Theorem 5.1(i):

$$
\mathrm{S}_{2}^{1} \vdash \square_{A, n}^{I} R_{0}^{N} \rightarrow\left(S \vee \square_{A, n} \perp\right) \text { and } \mathrm{S}_{2}^{1} \vdash\left(S \vee \square_{A, n} \perp\right)^{I} \rightarrow \square_{A, n} R_{0}^{N}
$$

We can plug in this last result in the reasoning of the proof of Theorem 6.1.

Thus, for a finitely axiomatized sequential $A, \mathbb{V}_{A}$ is closed under suprema. Moreover, every degree contains both a $\Sigma_{1}$ - and a $\Pi_{1}$-sentence over $A$ w.r.t. a chosen $N$.

We note that the suprema in $\mathbb{V}_{A}$ are sequential and hence cannot coincide with the suprema in $\mathbb{D}_{\text {all }}$, since, by a result of Pavel Pudlák ([Pud83]), sequential degrees

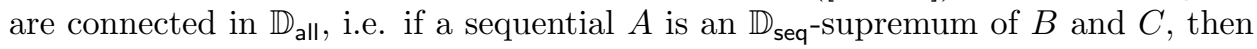
either $A \equiv B$ or $A \equiv C$.

Finally we note that our result fails if we try to extend it to the computably enumerable (c.e.) sequential case. E.g. $\mathrm{ACA}_{0} \triangleright \mathrm{PA}$, but for no extension $U$ of PA 
we have $\mathrm{ACA}_{0} \equiv U$, since such extensions are reflexive and no reflexive theory is mutually interpretable with a finitely axiomatized theory.

Remark 6.3. We remind the reader that AS is adjunctive set theory. By Theorem 6.1 , we find that the identical embedding in $\mathbb{V}_{\text {AS }}$ into $\mathbb{D}_{\text {seq }}$ is an isomorphism, since AS is in the minimal sequential degree. This theorem also follows from the result of Section 6 of [Vis08], which tells us that every sequential theory is definitionally equivalent (a.k.a. synonymous) with an extension of AS. This last result also works when we compare c.e. extensions of finite signature of AS to c.e. sequential theories of finite signature, and arbitrarily complex extensions of finite signature to arbitrarily complex sequential theories of finite signature.

We can lift Theorem 6.1 partly to the local interpretability degrees of sequential theories with no restrictions on the cardinality of the signature or on the complexity of the axiom set.

We remind the reader that $U$ locally interprets $V$ or $U \triangleright_{\text {loc }} V$, iff, for every finite subtheory $V_{0}$ of $V, U \triangleright V_{0}$. We write $\equiv_{\text {loc }}$ for the induced equivalence relation.

The degree of local interpretability of a theory $U$ with no restrictions on the cardinality of the signature and no restrictions on the complexity of the axioms set, is called a chapter in [MPS90].

Theorem 6.4. Let $U$ be any sequential theory, with no restrictions on the cardinality of the signature and no restrictions on the complexity of the axioms set. Let $A$ be finitely axiomatized and sequential with $A \triangleleft U$. Suppose $N: \mathrm{S}_{2}^{1} \triangleleft U$. Then there are sets of $\Sigma_{1}$-sentences $\mathcal{S}$ and of $\Pi_{1}$-sentences $\mathcal{P}$, such that:

$$
U \equiv_{\text {loc }}\left(A+\mathcal{S}^{N}\right) \equiv_{\text {loc }}\left(A+\mathcal{P}^{N}\right) .
$$

More precisely we can take $\mathcal{S}$ to consist of $\exists \Pi_{1}^{\mathrm{b}}$-sentences and $\mathcal{P}$ of $\forall \Sigma_{1}^{\mathrm{b}}$-sentences.

Proof. It is easily seen that there is a sequence $A=A_{0} \triangleleft A_{1} \triangleleft A_{2} \ldots$ of finitely axiomatized sequential theories such that $A_{i} \triangleleft U$ and, for any finitely axiomatized $B$ such that $B \triangleleft U$, there is an $i$ such that $B \triangleleft A_{i}$.

We define a sequence of $\Sigma_{1}$-sentences as follows. Let $S_{0}$ be $\top$. Suppose we already have that $\left(A+S_{0}^{N}, \ldots, S_{n}^{N}\right) \equiv A_{n}$ and, hence, $\left(A+S_{0}^{N}, \ldots, S_{n}^{N}\right) \triangleleft A_{n+1}$. Let $S_{n+1}$ be the sentence $R$ provided by Theorem 6.1 with $A+S_{0}^{N}, \ldots, S_{n}^{N}$ in the role of $A$ in the theorem, $N$ in the role of $N$ in the theorem and $A_{n+1}$ in the role of $B$ in the theorem. Clearly we find $\left(A+S_{0}^{N}, \ldots, S_{n+1}^{N}\right) \equiv A_{n+1}$. We take $\mathcal{S}:=\left\{S_{0}, S_{1}, \ldots\right\}$.

The $\Pi_{1}$-case is similar.

We see that this means that the embedding of the local degrees of arbitrary extensions of a finitely axiomatized sequential $A$ into the local degrees of arbitrary sequential theories is convex, and, ipso facto, a lattice homomorphism.

In Theorem 6.1 we showed that, for finitely axiomatized and sequential $A$ and $B$ with $A \triangleleft B$, we can find a sentence $C$ in the language of $A$ such that $(A \wedge C) \equiv$ $B$. This sentence can be chosen to be a $\Sigma_{1}$-sentence or a $\Pi_{1}$-sentence relativized to a fixed interpretation $N$ of $\mathrm{S}_{2}^{1}$ in $A$. E.g. if $A$ were GB we could take $N$ to be the finite von Neumann ordinals. Our construction has two disadvantages. 
First, the sentences used are metamathematical artifacts created using the FGH variant of the Rosser construction. Secondly, the conjunction of two such relativized sentences over $A$ does not seem to give us the supremum. In Theorem 6.5 , we repair these defects. Our sentences in Theorem 6.5 are restricted consistency statements relativized to a set of numbers that has a self-reference-free definition. Moreover, we have the desired supremum-conjunction correspondence. There is one cost: we cannot use any given interpretation $N$ of $\mathrm{S}_{2}^{1}$ but are forced to employ a special one. E.g., in the case of GB the result will not work for the finite von Neumann ordinals.

Theorem 6.5 uses Theorem 5.2 as the main technical lemma.

Theorem 6.5. Consider any finitely axiomatized sequential theory A. There is an $N_{0}: \mathrm{S}_{2}^{1} \triangleleft A$ such that, for all finitely axiomatized sequential theories $B_{0}, \ldots, B_{\ell-1}$, we have:

$$
\left(A \sqcup \bigsqcup_{j<\ell} B_{j}\right) \equiv\left(A+\left(\bigwedge_{j<\ell} \diamond_{B_{j}, \rho\left(B_{j}\right)}^{\top}\right)^{N_{0}}\right) .
$$

Proof. Let $A$ be finitely axiomatized and sequential. For any finitely axiomatized and sequential theories $B_{0}, \ldots, B_{\ell-1}$, we take $S:=\bigvee_{j<\ell} \square_{B_{j}, \rho\left(B_{j}\right)} \perp$. Note that there is a fixed $k$, such that $\rho(S) \leq k$, independent of the choice of the $B_{j}$. Let $N_{0}$ as given in Theorem 5.2 for $A$ and $k$. We find, for $m:=\max \left(\rho(A), k+\rho\left(N_{0}\right)\right)$,

$$
\mathrm{EA} \vdash \diamond_{A, m}\left(\bigwedge_{j<\ell} \diamond_{B_{j}, \rho\left(B_{j}\right)} \top\right)^{N_{0}} \leftrightarrow\left(\diamond_{A, \rho(A)} \top \wedge \bigwedge_{j<\ell} \diamond_{B_{j}, \rho\left(B_{j}\right)} \top\right) .
$$

It follows, by Theorems 3.4 and 3.5, that

$$
\begin{aligned}
A \sqcup \bigsqcup_{j<\ell} B_{j} & \equiv \mathrm{S}_{2}^{1}+\diamond_{A, \rho(A)} \top \wedge \bigwedge_{j<\ell} \diamond_{B_{j}, \rho\left(B_{j}\right)}{ }^{\top} \\
& \equiv \mathrm{S}_{2}^{1}+\diamond_{A, m}\left(\bigwedge_{j<\ell} \diamond_{B_{j}, \rho\left(B_{j}\right)}^{\top}\right)^{N_{0}} \\
& \equiv A+\left(\bigwedge_{j<\ell} \diamond_{B_{j}, \rho\left(B_{j}\right)} \top\right)^{N_{0}}
\end{aligned}
$$

We note that it follows that if $B \triangleright A$, then $B \equiv\left(A+\diamond_{B, \rho(B)}^{N_{0}} \top^{\top}\right)$.

Theorem 6.5 is clearly analogous to Theorem 3.4. The theorem shows that arbitrary finitely axiomatized sequential theories are in some respects very much like $\mathrm{S}_{2}^{1}$. Exploiting this analogy we can mimic the proof of Theorem 4.1 to obtain the following theorem.

Theorem 6.6. Let $A$ be finitely axiomatized and sequential. There is an $N_{0}: \mathrm{S}_{2}^{1} \triangleleft A$ and a function $\theta$ that sends finitely axiomatized sequential theories $B$ with $B \triangleright A$ to $\forall \Pi_{1}^{\mathrm{b}}$-sentences such that:

- if $B \triangleright C \triangleright A$, then $A+\theta(B)^{N_{0}} \vdash A+\theta(C)^{N_{0}}$.

- if $B \triangleright A$, then $B \equiv\left(A+\theta(B)^{N_{0}}\right)$.

The function $\Theta$ is recursive in an oracle for a $\Sigma_{1}$-complete set. 
Remark 6.7. We define $\mathbb{D}_{\text {seq }, A}$ as the degree structure of the finitely axiomatized sequential theories $B$ with $B \triangleright A$. Let the identical projection $\pi: \mathbb{P}_{A} \rightarrow \mathbb{D}_{\text {seq }, A}$ be given by $\pi(B):=B$. Let $\Theta: \mathbb{D}_{\text {seq }, A} \rightarrow \mathbb{P}_{A}$ be given by $\Theta(B):=A+\theta(B)^{N_{0}}$. When we think modulo the induced equivalence relations $-\Vdash$ and $\equiv$, Theorem 6.6 tells us that $\pi$ is a retraction, where $\Theta$ is the witnessing coretraction.

Open Question 6.8. Can we find, for any finitely axiomatized sequential theory $A$ and for any $N: \mathrm{S}_{2}^{1} \triangleleft A$, a mapping $B \mapsto C_{B}$ sending any finitely axiomatized sequential $B$ with $A \triangleleft B$ to an arithmetical sentence $C_{B}$, such that, for any finitely axiomatized sequential theories $B_{0}, \ldots, B_{n-1}$ with $B_{j} \triangleright A$ for al $j<n$, we have:

$$
\bigsqcup_{j<n} B_{j} \equiv\left(A+\bigwedge_{j<n} C_{B_{j}}^{N}\right)
$$

If so, can we get the $C_{B}$ to be $\Pi_{1}$ or $\Sigma_{1}$ ?

\section{The Case of $Q$}

Let $U$ be any theory of finite signature. We show that some extension $W$ of $\mathbf{Q}$ can function as the supremum of $\mathrm{Q}$ and $U$. We use the fact that $U$ is bi-interpretable and, hence, a fortiori, mutually interpretable with a theory, say $V$, of a binary predicate, say $R$. See [Hod93, Section 5.5]. We note that Hodges uses parameters. However these can be eliminated by raising the dimension and integrating them in our objects. It is sufficient to prove the desired result for $V$.

The theory $W$ in the language of $\mathrm{Q}$ is axiomatized by the following principles.

- $Q$

- $\exists ! a \forall x(x+a=a \wedge a+x=a)$. We call this unique $a$ : $\infty$. We define $\delta(x): \leftrightarrow \mathrm{S} x=x \wedge x \neq \infty$.

- $V^{\star}$, where $V^{\star}$ is the result of relativizing the quantifiers in $V$ to $\delta$ and replacing $R(x, y)$ by $x+y=\infty$.

Trivially we have $W \triangleright \mathrm{Q}$ and $W \triangleright V$. Suppose $K: Z \triangleright \mathrm{Q}$ and $M: Z \triangleright V$. We construct an interpretation $L: Z \triangleright W$. Since $J: \mathrm{Q} \triangleright(\mathrm{Q}+\forall x \mathbf{S} x \neq x)=: \mathrm{Q}^{*}$, for some $J$, we have $K^{\prime}=K \circ J: Z \triangleright \mathrm{Q}^{*}$.

We define an auxiliary theory $Y$ as follows the domain of $Y$ is partitioned in $\triangle_{0}$, $\triangle_{1}$ and $\{\infty\}$. We have $Q^{*}$ relativized to $\triangle_{0}$, and $V$ relativized to $\triangle_{1}$. We call addition in our copy of $\mathrm{Q}^{*}:+_{0}$, etc. The interpretability of $Y$ in $Z$ uses standard constructions on interpretations: how to make domains disjoint, how to conjure new objects out of the high hat, etc. ${ }^{14}$ We sketch briefly how this works in this specific case.

Suppose $K^{\prime}$ is $m$-dimensional and $M$ is $n$-dimensional, then we take $N$ to be $\max (m, n)+3$-dimensional. Let's assume that $m<n$. The case that $m \geq n$ is similar. An element $\vec{v}$ of $\delta_{K^{\prime}}$ reappears as $x, x, x, \vec{w}, \vec{v}$, where the length of $\vec{w}$ is $n-m$. The only role of $\vec{w}$ is to serve as padding. The elements $x, x, x, \vec{w}, \vec{v}$ give

\footnotetext{
${ }^{14}$ If we allow piece-wise interpretability these constructions are matter of course. But we can always execute them too by raising dimensions, provided that we have at least two objects in the domain.
} 
us the interpretation of $\triangle_{0}$. An element $\vec{v}$ of $\delta_{M}$ reappears as $x, x, y, \vec{v}$. These elements give us also the interpretation of $\triangle_{1}$. Finally, we define $\infty$ by the elements of the form $x, y, x, \vec{z}$, where $x \neq y$. The $\vec{z}$ is just padding. We define e.g.: $x_{0}, x_{1}, x_{2}, \vec{w}={ }_{N} y_{0}, y_{1}, y_{2}, \vec{z}$ iff $\left(x_{0}=x_{1}=x_{2}\right.$ and $y_{0}=y_{1}=y_{2}$ and $\vec{w}=\vec{w}_{0}, \vec{w}_{1}$ and $\vec{z}=\vec{z}_{0}, \vec{z}_{1}$ and $\delta_{K^{\prime}}\left(\vec{w}_{1}\right)$ and $\delta_{K^{\prime}}\left(\vec{z}_{1}\right)$ and $\left.\vec{w}_{1}=K_{K^{\prime}} \vec{z}_{1}\right)$ or $\left(x_{0}=x_{1} \neq x_{2}\right.$ and $y_{0}=y_{1} \neq y_{2}$ and $\delta_{M}(\vec{w})$ and $\delta_{M}(\vec{z})$ and $\left.\vec{w}={ }_{M} \vec{z}\right)$ or $\left(x_{0}=x_{2} \neq x_{1}\right.$ and $\left.y_{0}=y_{2} \neq y_{1}\right)$. We define the interpretations of the arithmetical operations and of $R$ in the obvious way.

We work in $Y$. We define arithmetical operations on the full domain, i.e. $\triangle_{0} \cup$ $\triangle_{1} \cup\{\infty\}$. We take as 0 the element $0_{0}$. We define: $\mathrm{S}(x):=\mathrm{S}_{0}(x)$ if $x \in \triangle_{0}$ and $\mathrm{S}(x):=x$, otherwise. We define addition as follows. In the diagram, $n$ ranges over $\triangle_{0}$ and $x$ ranges over $\triangle_{1}$. We define addition as follows.

\begin{tabular}{|c||c|c|c|}
\hline+ & $n$ & $y$ & $\infty$ \\
\hline \hline$m$ & $m+{ }_{0} n$ & $y$ & $\infty$ \\
\hline$x$ & $x$ & $\begin{array}{l}\infty \text { if } R(x, y) \\
y \text { otherwise }\end{array}$ & $\infty$ \\
\hline$\infty$ & $\infty$ & $\infty$ & $\infty$ \\
\hline
\end{tabular}

Here is the definition of multiplication:

\begin{tabular}{|c||c|c|c|c|c|}
\hline$\times$ & 0 & 1 & $n \neq 0,1$ & $y$ & $\infty$ \\
\hline \hline$m$ & 0 & $m$ & $m \times_{0} n$ & $\infty$ & $\infty$ \\
\hline$x$ & 0 & $x$ & $\begin{array}{c}\infty \text { if } R(x, x) \\
x \text { otherwise }\end{array}$ & $\infty$ & $\infty$ \\
\hline$\infty$ & 0 & $\infty$ & $\infty$ & $\infty$ & $\infty$ \\
\hline
\end{tabular}

It is easy to see that these tables give us the desired interpretation of $W$.

It is clear that the above result could have been proved in many other ways. For example we could have modified the theorem from [Hod93] in such a way that we can assume $R$ to be irreflexive and define $x+y=y$ if $R(x, y)$ and $x+y=x$ otherwise. In this way we can avoid the need for $\infty$.

The following result is immediate from the above considerations.

Theorem 7.1. The identical embedding of $\mathbb{V}_{\mathrm{Q}}$ into $\mathbb{D}_{\mathrm{all}}$ is convex, and, ipso facto, a lattice homomorphism.

Remark 7.2. We note that our result also works when we compare c.e. extensions of $Q$ of finite signature to c.e. theories of finite signature that interpret $Q$, and arbitrarily complex extensions of $Q$ of finite signature to arbitrarily complex theories of finite signature that interpret $Q$.

\section{List of Questions}

In this section we provide a list of open problems.

1. Can we find examples of $A$ such that $V_{A}$ does not have suprema, where $A$ is finitely axiomatized and $A \triangleright \mathrm{Q}$ ? In other words, what about the remaining cases of Švejdar's question? This question is already interesting if we drop the requirement that $A \triangleright \mathrm{Q}$ or if we replace $A$ by a c.e. theory of finite signature. 
2. Is there a recursive function $\Theta$ that maps the finitely axiomatized sequential theories to finitely axiomatized sequential theories of a given signature $\Sigma^{\star}$, such that (i) if $A \triangleleft B$, then $\Theta(A) \dashv \Theta(B)$ and (ii) $\Theta(A) \equiv A$. This is Open Question 4.6.

3. Can we find, for any finitely axiomatized sequential theory $A$ and for any $N$ : $\mathrm{S}_{2}^{1} \triangleleft A$, a mapping $B \mapsto C_{B}$ sending any finitely axiomatized sequential $B$ with $A \triangleleft B$ to an arithmetical sentence $C_{B}$, such that, for any finitely axiomatized sequential theories $B_{0}, \ldots, B_{n-1}$ with $B_{j} \triangleright A$ for all $j<n$, we have:

$$
\bigsqcup_{j<n} B_{j} \equiv\left(A+\bigwedge_{j<n} C_{B_{j}}^{N}\right)
$$

If so, can we get the $C_{B}$ to be $\Pi_{1}$ or $\Sigma_{1}$ ? This is Open Question 6.8.

\section{REFERENCES}

[Bus11] S.R. Buss. Cut elimination in situ. http://math.ucsd.edu/ sbuss/, 2011.

[Fri07] H. Friedman. Interpretations according to Tarski. This is one of the 2007 Tarski Lectures at Berkeley. The lecture is available at http://www.math.osu.edu/ friedman.8/pdf/Tarski1,052407.pdf, 2007.

[Ger03] Philipp Gerhardy. Refined Complexity Analysis of Cut Elimination. In Matthias Baaz and Johann Makovsky, editors, Proceedings of the 17th International Workshop CSL 2003, volume 2803 of LNCS, pages 212-225. Springer-Verlag, Berlin, 2003.

[Ger05] Philipp Gerhardy. The Role of Quantifier Alternations in Cut Elimination. Notre Dame Journal of Formal Logic, 46, no. 2:165-171, 2005.

[Hod93] W. Hodges. Model theory. Encyclopedia of Mathematics and its Applications, vol. 42. Cambridge University Press, Cambridge, 1993.

[HP91] P. Hájek and P. Pudlák. Metamathematics of First-Order Arithmetic. Perspectives in Mathematical Logic. Springer, Berlin, 1991.

[Kra87] J. Krajíček. A note on proofs of falsehood. Archiv für Mathematische Logik und Grundlagenforschung, 26:169-176, 1987.

[LS08] P. Lindström and V. Yu. Shavrukov. The $\forall \exists$ theory of Peano $\Sigma_{1}$ sentences. Journal of Mathematical Logic, 8(2):251-280, 2008.

[MPS90] J. Mycielski, P. Pudlák, and A.S. Stern. A lattice of chapters of mathematics (interpretations between theorems), volume 426 of Memoirs of the American Mathematical Society. AMS, Providence, Rhode Island, 1990.

[Pud83] P. Pudlák. Some prime elements in the lattice of interpretability types. Transactions of the American Mathematical Society, 280:255-275, 1983.

[Pud85] P. Pudlák. Cuts, consistency statements and interpretations. The Journal of Symbolic Logic, 50:423-441, 1985.

[Sha10] V.Yu. Shavrukov. Effectively inseparable Boolean algebras in lattices of sentences. Archive for Mathematical Logic, 49(1):69-89, 2010.

[Smo85] C. Smoryński. Nonstandard models and related developments. In L.A. Harrington, M.D. Morley, A. Scedrov, and S.G. Simpson, editors, Harvey Friedman's Research on the Foundations of Mathematics, pages 179-229. North Holland, Amsterdam, 1985.

[Ste89] A.S. Stern. Sequential theories and infinite distributivity in the lattice of chapters. The Journal of Symbolic Logic, 54:190-206, 1989.

[Šve78] V. Švejdar. Degrees of interpretability. Commentationes Mathematicae Universitatis Carolinae, 19:789-813, 1978.

[Vis92] A. Visser. An inside view of EXP. The Journal of Symbolic Logic, 57:131-165, 1992.

[Vis93] A. Visser. The unprovability of small inconsistency. Archive for Mathematical Logic, 32:275-298, 1993.

[Vis05] A. Visser. Faith \& Falsity: a study of faithful interpretations and false $\Sigma_{1}^{0}$-sentences. Annals of Pure and Applied Logic, 131:103-131, 2005.

[Vis08] A. Visser. Pairs, sets and sequences in first order theories. Archive for Mathematical Logic, 47(4):299-326, 2008. 
[Vis09] A. Visser. Cardinal arithmetic in the style of baron von Münchhausen. Review of Symbolic Logic, 2(3):570-589, 2009. doi: 10.1017/S1755020309090261.

[Vis10] A. Visser. What is the right notion of sequentiality? Logic Group Preprint Series 288, Department of Philosophy, Utrecht University, Heidelberglaan 8, 3584 CS Utrecht, http://www.phil.uu.nl/preprints/lgps/, 2010.

[Vis11] A. Visser. Can we make the Second Incompleteness Theorem coordinate free. Journal of Logic and Computation, 21(4):543-560, 2011. First published online August 12, 2009, doi: $10.1093 / \log c o m /$ exp048.

[Vis12] A. Visser. The arithmetics of a theory. Logic Group Preprint Series 293, Department of Philosophy, Utrecht University, Heidelberglaan 8, 3584 CS Utrecht, http://www.phil.uu.nl/preprints/lgps/, 2012.

[Wil86] A.J. Wilkie. On sentences interpretable in systems of arithmetic. In Logic Colloquium '84, volume 120 of Studies in Logic and the Foundations of Mathematics, pages 329-342. Elsevier, 1986.

[WP87] A. Wilkie and J.B. Paris. On the scheme of of induction for bounded arithmetic formulas. Annals of Pure and Applied Logic, 35:261-302, 1987.

Department of Philosophy, Utrecht University, Janskerkhof 13A, 3512Bl Utrecht, The NETHERLANDS

E-mail address: albert.visser@phil.uu.nl 